\title{
羽rogress in Attedirine.
}

\section{REPORT ON DISEASES OF THE NERVOUS SYSTEM.}

By Jayes J. Putnam, M.D.

\section{Pathology of the Cortex Cereari.}

Since the publication of the highly important experiments upon the localization of centres for muscular movements in the cortex cerebri, made first by Fritsch and Hitzig, of Berlin, afterwards with, in the main, similar results, by Nothnagel, of Berlin, Ferrier, of London, and others (vide this JounnaL for July 24 and 30, 1873, and April 30, 1874), a number of clinical cases have been reported, in which defined lesions of the cortex produced muscular convulsions, with subsequent partial paralysis, so localized as to strikingly confirm the justice of applying to man, in principle, at least, the results obtained with the brains of animals (Archiv für Psychiatrie u. Nervenkr. 1872, Bd. iii. caso by Hitzig; Virchow's Archiv, Bd. lvi., case by Wernher; Med. Times and Gaz., Nov. 30, 1872, cases by Hughlings Jackson ; also Arch. für Psychiatrie, Bd. iv., 3 cases by Bernhardt). Among these should be classed a case reported by Bartholow (Am. Journal of the Med. Sci., April, 1874), who, having a patient in whom the dura mater over the upper surface of the brain had been extensively laid bare by an epithelioma of the bone, thrust needles to different depths into the brain, and passed a current of electricity between them, with the effect of causing contraction of the muscles of the neck, with the extensors of the arm and leg, both on the opposite side of the body. An epileptiform,attack, limited to the arm, shoulder and neck of the opposite side, occurred, also, after an injury of some severity to a limited region of the cortex.

Theso experiments and cases, taken in connection with the largo number of instances where aphasia has been associated with discase of the convolutions about the fissure of Sylvius,* together with the presumptive evidence furnished by anatomy, would seem unquestionably to teach that the different muscles of the body are represented in cortain regions of the cortex cerebri, constituting centres, from the irritation of which the corresponding muscles can be excited to contraction, although, to judge from pathological observation, the arrangement of these cerebral centres may not be, for every individual, precisely the same.

This view has, however, been combatted, especially by Dr. BrownSéquard, $\nmid$ as has been already stated in this Jounsal (loc. cit.), who brings forward a large number of cases to prove that diseaso may exist in any part of the brain without causing symptoms at all, or only on the same side of the body with the lesion, and conversely, that disease in almost any part of the brain may produce almost any symptom. From these, and other similar facts, he concludes that the theory which assigns special functions to the different centres in the brain, especially the cortex cerebri, and explains symptoms by the direct irrita-

- For summaries of these cases, vide papers by Lohmeyer, in the Arch. far Klin. Chi. rurgle, and Men\%el, in the Wiener Med. Woehenschr., I874.

$t$ Vire, anong other papers, the Archives of Scientilic and Practical Medicine for January, February and March, 1873. 
tion or destruction of those centres, is absolutely untenable, and proposes the theory instead, that the apparent lesion acts indireclly upon "distant centres," exerting over them an irritating or a paralyzing (inhibitory) influence, just as peripheral lesions are known sometimes to do. In this way, alone, he believes, are symptoms of disease in the cerebral lobes, the cerebellum, and its peduncles produced, and, in part, those of disease of the base of the brain.

Though never to the same extent, this indirect influence of cerebral lesions has already been recognized by many observers,* but it is also widely questioned whether the principle of the possible assumption by one part of the brain of the functions of another part which may have been destroyed, cannot be so extended as to explain many of the anomalous cases referred to, leaving the theory of the direct influence of lesions, for which the positive evidence is so strong, otherwise intact.

This principle is well recognized in general pathology, and, in view of the intimate connections existing between different parts of the ganglionic masses of the brain, particularly the cortex cerebri, its influence in cerebral pathology might fairly bo looked for, especially if we admit that the nerve-cells, of the cortex for example, are less distinguished from one another by any physiological peculiarities than by their education, i. e., by the excitations which they habitually receive and transmit.

There appears, moreover, to be the objection to Dr. Brown-Séquard's argument, that, while specifically disproving the existence of "centres," even at the base of the brain, he really presupposes their existence somewhere, as, indeed, the occurrence of definite symptoms, such as we observe clinically, whether produced directly or indirectly, demands, and it seems to be necessary in either case to admit the validity of the principle of vicarious functions in one form or another.

The positive evidence for the existence of centres, in the modified sense, as regards the cortex cerebri, has already been alluded to.

Dr. Ilughlings Jackson, whose observations have several times boen mentioned in these Reports (loc. cit.), insists on the importance of studying them rather through the careful observation of unilateral convulsions beginning in special muscles, or muscular groups, than by the class iof paralyses, for the reason stated, that from absence of paralysis we caunot confidently infer the presence of corresponding centres in the cortex, while from convulsion we can infer their presence and their excitation.

According to his, and others', view of cerebral physiology, we can already say, in general terms, that the whole muscular system of the body is probably represented several times within the central nervous system, in different masses of gray matter, that of the spinal cord, of the cerebral ganglia (excopt, perhaps, the optic thalamus), of the ccrebellum, and of the cortex cerebri, from any one of which it is capable of being excited, entirely or in part, to functional activity. Among these ganglionic masses, it is the cortex cerebri which is so placed as to receive the greatest number and variety of nervous impressions through the channels of the senses, which it also has the power of storing up for future use ; and there also the greatest variety and number of voluntary impulses naturally have their origin, the active vol-

" Vide, for example, Jaccoud, Path. Interne, 4th ed., i. p. 286. 
untary impulse (i. e. the nervous impression which excites the nervecentres in which it has its seat so strongly that through them muscular action is called out) being the resultant of all the impulses, or impressions, present at any given moment in the brain.

The exclusive employment, then, of single muscles, or of a number of muscles in new, or comparatively new, combinations for definite purposes, is, according to this viow, a function (speaking physiologically) of the cortex cerebri.

Less highly differentiated, or complicated, i. e., more antomatic, movements or even complicated ones, if they have become familiar through repetition, may be called out through the influence of the cerebral ganglia, the cerebellum and the spinal cord. The experiments of Hitzig and Ferrier plainly afford striking support to these views.*

Hitzig, and still more Ferrier, found, moreover, that when the irritation of a definite point of the cortex cerebri, with induction currents, was continued for a certain time, epileptiform convulsions of the muscles on the opposite side of the body could be induced, beginning in the muscles corresponding to the point irritated. It was found also, by both IHitzig und Ferrier, that when any motor centre was cut out, the animals were not paralyzed for the more or less automatic movements of walking or running, but that they would allow the corresponding member to be placed in awkward positions without attempting to correct them, indicating an impairment of muscular sense, although general sensation seemed unimpaired.

Applying these views to pathological cases, we can, according to Hughlings Jackson, assume that localized unilateral convulsions, or convulsions beginning as such, even if they spread afterwards to other parts of the body, are due to irritations centring at definite points of the cortex cerebri.

Such convulsions occur consequent on the nutritive changes due to embolic plugging of arteries, to syphilitic and other tumors, or to localized meningitis, if they cause irritation of the cortex cerebri at certain points (vide Medical Times and Gazette, 1873 and 1874, papers by Jackson and Broadbent), and Jackson considers it probable that many cases now classed under the head of epilepsy (a namo which he thinks should not be used to designate a collection of symptoms, but a condition of mal-nutrition of nervous centres, which renders them liable to periodic outbreaks of excitement, and which may affect many different parts of the brain) are due to lesions there, although these lesions may easily escape notice, causing, as they do, irritation only, not destruction, of the parts involved. Convulsions of this kind may be followed by more or less temporary, incomplete paralysis, or loss of acquired dexterity, of the parts attacked, of which temporary aphasia is a type. (Speech, unless simply ejaculatory, occurs when the excitations, of which the subjective side is thought, excite the motor centres for the organs of articulation, \&c., so strongly that muscular contraction results, and in the conception of words without their utter-

- The criticism made by Brown-Séquard and others that, in these experiments, it is the irritation, not of the cortex, but of ganglia lying beneath it, which causes tho movements, is discussed in this JounNal for July 16, 1874 .

t Compare Reports on Mental Discuses in this Jounsal for July 31, 1873. Also, West Riding Asylum Reports, article Epilepsy. Hughlings Jackson believes, on theoretical grounds, that the spreading takes place through the indirect influence of the arterial districts.

VoL. XCI. No. 5B 
ance, the same centres are implicated, as Hughlings Jackson believes, but less strongly excited.)

Such temporary paralysis may, perhaps, be due to exhaustion of the nervous centres, consequent on their over-action.

When the paralysis is more extensive and complete, as in most cases of cerebral hemiplegia from hæmorrhage, the corpus striatum, or motor tract connected with it, is generally the seat of the lesion, and the paralysis is not necessarily preceded by convulsion, as is almost invariably the case when the cortex is affected.

\section{Shock and Cerebral Concussion.}

Our knowledge of these somewhat obscure affections has received some valuable additions at the hands of the experimental physiologist, within the past few years, as is clearly shown in some excellent papers by Prof. Fischer, of Breslau,* and Brunton, of London.t

The characteristic symptoms of shock are stated as :- pallor and coldness of the skin, weakness and irregularity of the pulse, oppressed and sighing character of the respiration, nausea and romiting, and apathy of mind without entire loss of consciousness, in brief, a choleralike prostration; its causes as: painful impressions on the sensitive nerves (as in large burns), injuries to bones, and, above all, injuries to the abdominal viscera and the genitals.

Besides this form there is another, called by Travers "prostration with excitement," of which we will only say here that, according to Fischer, it is generally secondary to the form described.

Shock has been pathologically defined by Savory as " the paralyzing influence of a sudden and violent injury to nerves on the activity of the heart." According to Brunton, this probably occurs in many cases at the outset at least, but the important agency, to which especially the continuance of the symptoms is due, is believed to be reflex paralysis of the vascular system of the whole body, but particularly of the abdominal cavity, due to the inhibitory influence which the injury exerts upon the vaso-motor centres, which lie mainly in the medulla oblongata.

That such a reflex paralysis is physiologically possible is an acknowledged fact, and the experiments of $v$. Bezold and Bever, and others, have shown also that the vascular, especially the venous, system of the abdomen, supplied by the splanchnic nerves, may dilate, when those nerves are cut, so much as to contain nearly all the blood of the body, which stagnates there as in a reservoir. It is especially upon the experiments of Goltz, now of Strasburg, however, that these pathological views are based, who found that a number of light, successive blows upon the abdomen of a frogt caused a temporary standstill of the heart; and, further, that after it had begun again to beat, it received and sent out but a small quantity of blood, indeed none at all if the frog was held upright, while examination showed that the missing blood had collected, as described, in the veins of the abdomen.

Goltz at first believed that the paralysis affected solely the vessels of the irritated part, the abdomen, but it was found to be universal, and producible, moreover, by a jar of the whole body.

\footnotetext{
- Volkmann's Sammiung Klinische Vortrage, Nos. 10 and 27.

+ Practitioner, October, 1873 .
\pm Brown-Séquard reports that he had made similar observations upon other animals still earlier.-RBPORTE⿱.
} 
Through this rapid abstraction of blood, and consequent failure of the circulation, it is claimed that the pallor of the skin, rapid fall of temperature of the body and cerebral symptoms are readily explained. When recovery takes place, it is rather gradual, since the veins contract again but slowly, more slowly than the small arteries; and this, as Brunton believes, gives us a suggestion as to the pathology of fainting (syncope), of which the symptoms strongly resemble those of shock.

In the treatment of shock, there are two therapeutical indications: to cause the vessels to contract, and to stimulate the heart's action, besides, of course, keeping up the temperature. Stimulants, ammonia held to the nose, excitation of the skin, are all agents recognized experimentally and clinically, as useful for these ends, and Brunton recommends further, on theoretical grounds, the giving of digitalis in large doses, as has, he says, indeed, been done by Wilks* (half a drachm of the tincture every hour for seven doses), with excellent results, in one case where other means had failed.

The pathology of cerebral concussion is believed by Fischer, reasoning by exclusion and analogy, and from the results of autopsies, to be analogous to that of shock, except that the reflex paralysis (in an uncomplicated case, which is a rarity) affects the vessels of the brain alone. He points out that dilatation of the vessels so interferes with the circulation that the tissues are no longer properly nourished, and are virtually in a state of anæmia, which accounts for the coma, and, acting on the heart through the vagus nerve (for whose nucleus in the medulla oblongata the absence of well-oxydized blood is known to constitute an irritant), also for the slow, irregular pulse-the two characteristic symptoms of that state. Shock and concussion are often followed by symptoms which belong, in general, rather to the direct injuries to the central organs or their membranes which so often occur, but there is some reason to believe that the periodic outbreak of nervous symptoms which are often observed in the sequel (such as epilepsy), may sometimes be the indication of a morbid habit which was instituted in the original attack.

Ar a recent meeting of the Obstetrical Society of Edinburgh, Dr. Cuthbert showed a rare and interesting case of strangulation in utero. The mother of several healthy children miscarried for the second time March 29, 1874 . Her last menstruation was on Dec 17, 1873; consequently, the fatus was about eight and a half months old. It was perfectly healthy and well developed. No reason could be assigned by the patient for the accident. On examining the foetus, the funis was found coiled twice round the neck, and under the coiling it was lightly tied in a single knot. The part of the funis from the umbilicus to the neck was completely on the stretch, and its whole length from the insertion in the placenta to the umbilicus was about seventeen inches, or two and one half times the length of the foetus. The part continuous with the fotal circulation was round, and of a natural size; but the other part, viz., from the neck of tho foetus to the placenta, was small, and cord-like.

There can be no doubt that the death of the foetus, and, consequently, the miscarriage, was caused by the tying and coiling of the funis round the neck. Probably, it was also hastened, as the fotus grew larger, by putting the smaller part of the cord on the stretch, which would tend to tighten the knot and coils; at the same time it would also stop the circulation.-Edinburgh Medical Journal, June, 1874.

\footnotetext{
- Medical Times and Gazette, Jan. 16, 1864.
} 